\title{
Desarrollo de un modelo para la gestión del humedal Urama, Venezuela
}

\author{
Development of a model for the management \\ of the Urama wetland, Venezuela
}

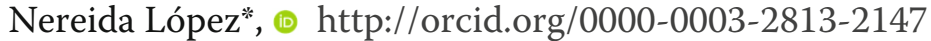 \\ y Edilberto Guevara-Pérez** ๑ https://orcid.org/0000-0002-0192-2176
}

Citar este artículo como: López, N. y Guevara Pérez, E. (2020). Desarrollo de un modelo para la gestión del humedal Urama, Venezuela. Revista Nodo, 15(29), pp. 38-55.

\section{Resumen}

El concepto de gestión se ha integrado últimamente a la ecología como un proceso planificado, dinámico y continuo. El compromiso ecológico global establece metas de protección ambiental implementando áreas protegidas (AP). La planificación de la gestión consiste en definir los objetivos respecto de las características ecológicas, procesos y valores del ecosistema. El objetivo de la investigación es la de desarrollar un modelo para la gestión del humedal Urama, (HU), Venezuela, basado en el análisis de las políticas y estrategias de gestión de humedales de 14 países de América Latina y España. El modelo aplica la valoración de los componentes de la Convención de Ramsar: política, características ecológicas, evaluación, objetivos, y plan de acción. Como resultado se ha obtenido un Indicador de Modelo de Gestión de Humedales (IMGH), cuyo valor cuantitativo integral es 0,67, siendo el máximo igual a 1. Para la valoración cualitativa del IMGH, 13,3 \% alcanza la categoría "muy alto", 33,33\% "alto", 20\% "mediano" y $33,33 \%$ "bajo". Con este resultado se desarrolla un modelo para la gestión del $\mathrm{HU}$ integrando 5 componentes, 28 variables y 56 atributos para un proceso de diagnóstico y evaluación, considerando las variables de impacto para la toma de decisión del Plan de Acción.

Palabras clave: Ecosistema, Ecológicas, Estrategias, Gestión ambiental, Evaluación, Humedal.

\section{Abstract}

The concept of management has recently been integrated into ecology as a planned, dynamic and continuous process. The global ecological commitment establishes environmental protection goals by implementing protected areas (PAs). Management planning consists of defining the objectives with respect to the ecological characteristics, processes and values of the ecosystem. The objective of the research is to develop a model for the management

Fecha de recepción: 25 de mayo de 2020 • Fecha de aceptación: 30 de julio de 2020

\footnotetext{
* Magíster en Ingeniería Ambiental. Investigadora del Centro de Investigaciones Hidrológicas y Ambientales (CIHAM-UC). Universidad de Carabobo, Valencia, Venezuela. Correo electrónico: nereidalopezster@gmail.com

*: Posdoctorado en Gestión de Recursos Hídricos. Profesor emérito de Ingeniería Civil y Ambiental. Centro de Investigaciones Hidrológicas y Ambientales (CIHAM-UC). Universidad de Carabobo, Valencia, Venezuela. Correo electrónico: eguevara@ uc.edu.ve; eguevara99@gmail.com
} 
of the Urama wetland (HU), Venezuela, based on the analysis of the wetland management policies and strategies of 14 Latin American countries plus Spain. The model applies the assessment of the components of the Ramsar Convention: Policy, ecological character, assessment, objectives, and action plan. As a result, a Wetland Management Model Indicator (WMMI) has been obtained, whose integral quantitative value is 0.67 , the maximum being equal to 1 . For the qualitative valuation of the WMMI, 13.3\% reaches the category "very high", 33.33\% "high", 20\% "medium" and 33.33\% "low". With this result, a model for HU management is developed, integrating 5 components, 28 variables and 56 attributes for a diagnosis and evaluation process, considering the impact variables for the decision making of the Action Plan.

Keywords: Ecosystem, Ecological, Strategies, Environmental management, Assessment, Wetland.

\section{Introducción}

Los humedales constituyen una amplia variedad de ecosistemas caracterizados por presencia predominante de agua y sustrato inundado durante gran parte del año. Se definen como sistemas transicionales entre terrestres y acuáticos (Cowardin, Carter, Golet y Laroe, 1979, p. 3); siendo sus principales indicadores de humedad la composición de la comunidad vegetal, la morfología del suelo y la hidrología del sitio (Federal Geographic Data Committee, FGDC, 2013, p. 7).

La definición de humedales se centra en la dinámica de los procesos hidrológicos de precipitación, escorrentía y volumen de agua almacenada (Hammer, 1986, p. 1951) y el reconocimiento preliminar para determinar el cambio temporal de los sitios de humedales se basa en las características hidrológicas similares y diferentes edades del suelo (Noon, 1996, p. 102).

Los humedales de extremo seco son los más abundantes en las cuencas fluviales e incluyen: bosques ribereños, límites entre tierras altas y humedales depresivos, y conectividad entre hábitats, un componente importante para mantener la biodiversidad y la fragmentación de paisajes (Whigham, 1999, p. 34).

Las características de los humedales están representadas por la densidad y el aislamiento de los mismos. La variabilidad en forma, tamaño y dispersión espacial se observan en mapas digitales que permiten examinar los efectos humanos en las métricas de mosaico correspondientes (Gibbs, 2000, p. 314).

La existencia de capital natural y los servicios de los sistemas ecológicos contribuyen al bienestar humano y por lo tanto representan parte del valor económico total del planeta (Costanza et al., 1997, p. 253). Es por ello que la identificación y valoración de los servicios ecológicos requiere de planificación territorial y la necesidad de lograr un manejo sustentable de los recursos naturales.

El término gestión tiene un origen económico y describe el ciclo de planificación, realización, control y adaptación. El plan de gestión para un ecosistema es parte de un proceso de gestión planificada dinámica y continua, teniendo en cuenta los resultados de seguimiento, como un proceso a largo plazo (Gattenlöhner, Hammerl-Resch y Jantschke, 2004, p. 17).

Las Áreas Protegidas (AP) se han convertido en una de las estrategias de manejo para la conservación de la biodiversidad, como modelo de la asociación del ser humano con la naturaleza (COMTEMAOLACEFS, 2015, p. 31). Las instituciones que gestionan las AP deben disponer de la información, personal, fondos, capacidad organizativa y nuevas tecnologías, como Sistemas de Información Geográfica (SIG), para usarlas como herramientas de planificación (Kugler, 2003, p. 20).

En el siglo XXI, la gestión de humedales ha venido introduciendo como criterio el procesamiento de imágenes satelitales, incorporando conceptos de heterogeneidad, fragmentación, conectividad y barreras al movimiento biológico (Euliss, Smith, Wilcox y Browne, 2008, p. 560). Un ejemplo son los ajustes de límites y el cambio de uso de la tierra y cobertura de la tierra (UTCT) en la Reserva 
Natural Nacional Paleocoast de Tianjin, China, y su humedal, donde el desarrollo económico local es la principal fuerza impulsora de UTCT, han proporcionado un modelo para equilibrar la protección y el desarrollo sostenible de los humedales costeros (Xie, Xu, Duan y Xu, 2012, p. 56). Sin embargo, el marco para la acción nacional y la cooperación internacional en pro de la conservación y el uso racional de los humedales lo establece la Convención sobre Humedales (Secretaría de la Convención de Ramsar, 2017, p. 1) y como tal, reconoce que la designación de humedales de importancia internacional (Sitios Ramsar, SR) establece el punto de partida para asegurar la sostenibilidad y el mantenimiento de los servicios de los ecosistemas, mediante un proceso de planificación con la participación de todos los interesados directos (Secretaría de la Convención de Ramsar, 2010, p. 6).

Los criterios de la Convención de Ramsar, han variado con la Conferencia de las Partes, (COP). Desde la COP5 (1993) a la COP10 (2008) se han adoptado los lineamientos para la planificación de los SR y otros humedales, asi como la descripción de las características ecológicas y el uso racional se equiparó al uso sostenible (Secretaría de la Convención de Ramsar 2010, p.6). La última COP (2018) adoptó entre otras orientaciones, identificar y restaurar turberas como SR para la regulación del cambio climático mundial (Secretaría de Ramsar, 2018, p. 1).

Venezuela como país firmante de la Convención de Ramsar, ha definido 5 áreas Ramsar con una superficie de 265,668 hectáreas; sin embargo, aunque en la década de los años 90 del siglo XX, las políticas implementadas fueron efectivas para establecer inventarios de humedales con indicadores positivos hacia la sostenibilidad, como un número significativo de humedales relevantes (Rodríguez, 1999, p. 11), en el presente año no se ha desarrollado la actualización de inventarios, además no cuenta con una política y estrategia de manejo de humedales.

Debido a la necesidad e importancia de gestionar los humedales ante los impactos introducidos por los sectores claves (producción del agua, energía, minería, agricultura, turismo, industria, silvicul- tura, acuicultura, pesca) que generan cambios de las características ecológicas debido a los cambios UTCT, en el Centro de Investigaciones Hidrológicas y Ambientales de la Universidad de Carabobo (CIHAM-UC) se ha emprendido esta investigación con el objetivo de desarrollar un modelo para la gestión del humedal Urama, que proporcione un instrumento complementario de planificación y contribuya a la sostenibilidad de los humedales sobre la base del enfoque por ecosistema, introduciendo un eje transversal de variables de impacto para la evaluación de los humedales, lo cual permitirá definir y delimitar las unidades de ordenamiento territorial del humedal como AP.

El alcance del análisis de las Políticas y Estrategias de Manejo de Humedales (PEMHs) parte del contexto internacional y nacional de catorce (14) países de América Latina (AL) y España, sobre la base del Modelo de Humedales de la Convención de Ramsar (MHCR) (Secretaría de Ramsar, 2010, p. 37), en una matriz multicriterio (MMC), valorando los componentes y criterios que contienen. El resultado define la formulación de la estructura de un modelo para la gestión del humedal Urama.

\section{Materiales y métodos}

El diseño de la investigación es de tipo documental, basado en la bibliografía referenciada sobre modelos de manejo de humedales de América Latina y España, aportaciones científicas y los datos elaborados por las fuentes oficiales sobre las PEMHs e instrumentos complementarios como planes ambientales e inventarios de humedales, que serán analizadas conforme a la estructura de manejo correspondientes al MHCR.

Las PEMHs comprenden 14 modelos de manejo de humedales de América Latina (Argentina, Brasil, Bolivia, Chile, Colombia, Costa Rica, Ecuador, Honduras, México, Panamá, Paraguay, Perú, Uruguay y Venezuela) y el modelo de gestión de España, que se aplican oficialmente a todos los humedales de cada país seleccionado, las cuales serán analizadas identificando componentes, variables y atributos que se encuentren establecidas en 
el MHCR, mediante la metodología de una MMC, asignando valores cuantitativos que determinen la existencia de la variable y atributo en un rango de categorías desde muy bajo a muy alto.

Para Venezuela, los documentos de análisis consistieron en el Plan Nacional Ambiental 2012-2030, Plan Estratégico de Áreas Protegidas 2013-2019 y Estrategia Nacional de Diversidad Biológica 2010-2030.

\section{Área de Estudio}

La cuenca de Urama se encuentra ubicada en la Región Centro Norte Costera, Estado Carabobo, formada por el aporte de los ríos Temerla, Canoabo, Sanguiguela y Alpargatón, En la planicie aluvial al norte se da lugar el humedal Urama para descargar en el mar Caribe (Figura 1).

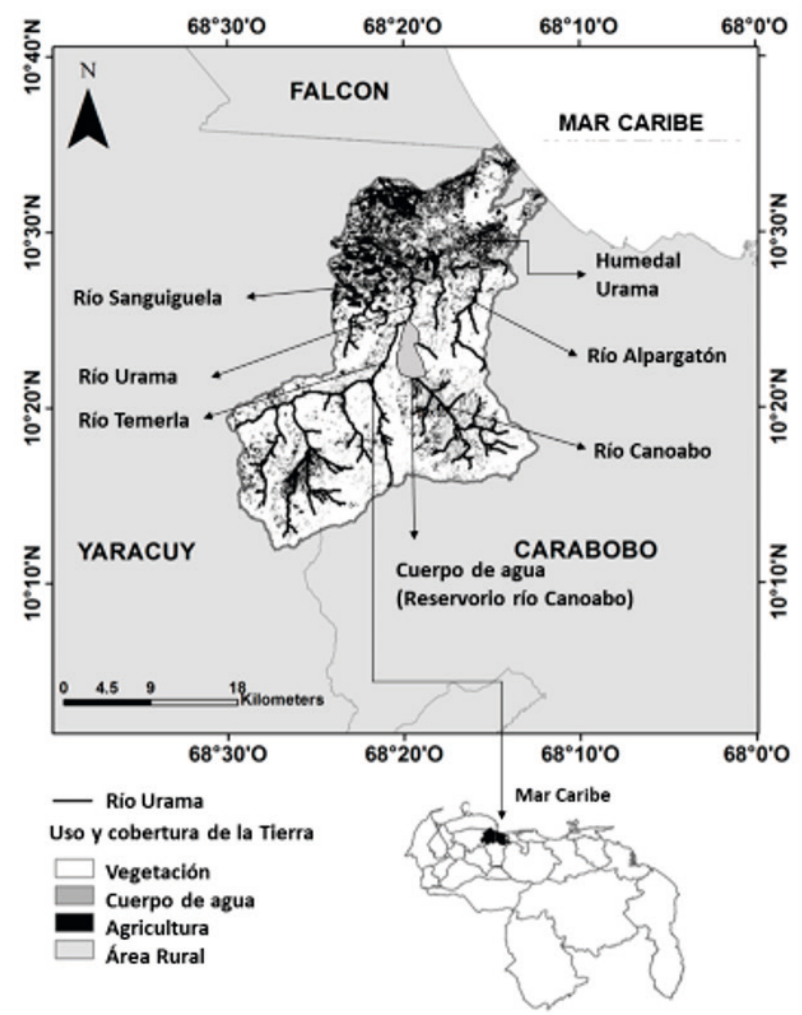

Figura 1. Mapa de ubicación del área de estudio: cuenca del río Urama, Carabobo, Venezuela. Fuente: Imagen obtenida del Sistema de Información Geográfico ArcGIS (2017).
La cuenca comprende 31.715 ha, es una importante fuente de producción de agua mediante un reservorio proveniente del río Canoabo, ubicado en la cuenca media. Se caracteriza además por los UTCT clasificado en áreas de vegetación, cuerpo de agua, actividad agropecuaria, rural y áreas deforestadas (PEQUIVEN, 2014, p. 289).

\section{Análisis de las PEMH de América Latina y España}

La metodología consiste en la definición y elaboración de una $M M C$ integrada que permita cuantificar la valoración de los componentes de la MHCR; se basa en modelos de decisión que contienen posibles soluciones que requieren ser evaluadas, según las unidades y un conjunto de medidas de desempeño respecto a cada uno de los criterios (Galarza, 2011, p. 12).

La estructura del MHCR es la base para realizar la comparación de las opciones de gestión de 15 países (Argentina, Brasil, Bolivia, Chile, Colombia, Costa Rica, Ecuador, España, Honduras, México, Panamá, Paraguay, Perú, Uruguay y Venezuela). Se divide en 5 componentes, 25 variables y 56 atributos: 1. Política; 2. Descripción de componentes ambientales, (Tabla 1); 3. Evaluación; 4. Objetivos y 5. Plan de acción, (Tabla 2), (Secretaría de la Convención de Ramsar, 2010, p. 14).

La MMC está representada por una matriz de evaluación $x$ de $n$ opciones de decisión y $m$ criterio o variables. El puntaje de desempeño bruto para la opción de decisión $i$ con respecto al atributo $j$ es denotado por $x m i j$. La importancia de cada criterio se da en unos pesos dimensionales vector $W$ que contiene $w$ pesos, donde $w j i$ denota el peso asignado al j-ésimo atributo del criterio $m$ (Hajkowicz y Collins, 2007, p. 1554). El instrumento también se referencia en dos metodologías reconocidas internacionalmente: "Evaluación rápida" y "Priorización del manejo de áreas protegidas y herramienta de seguimiento de efectividad de la gestión" (COMTEMA-OLACEFS, 2015, p. 8). 
Tabla 1. Matriz multicriterio para humedales de la Convención de Ramsar (componentes 1 y 2)

\begin{tabular}{|c|c|}
\hline Componentes X & Variables $(\mathbf{m})$ \\
\hline 1. Política & 1. Instrumento jurídico de creació \\
\hline \multirow{2}{*}{$\begin{array}{l}\text { 2. Descripción } \\
\text { características } \\
\text { ecológicas }\end{array}$} & 2. Geomorfología \\
\hline & 3. Geología / suelos \\
\hline \multirow{2}{*}{$\begin{array}{l}2.1 \text { Componentes } \\
\text { ecológicos, } \\
\text { socioeconómicos y } \\
\text { culturales }\end{array}$} & 4. Clima \\
\hline & 5. Hidrografía \\
\hline
\end{tabular}

6. Ecosistemas / hábitats

7.Vegetación

8. Fauna

9. Socioeconómicos

10. Culturales

Sub total valor 1 y 2.1

$\begin{array}{ll}\begin{array}{l}2.2 \text { Procesos } \\ \text { ecológicos }\end{array} & \text { 11. Ecosistemas / hábitats }\end{array}$

12. Vegetación / fauna

2.3 Servicios de 13. Aprovisionamiento ecosistemas

14. Regulación

15. Socioeconómico-cultural

Subtotal valor 2.2 y 2.3
Atributos (j)

Ramsar (i)

Política, estrategias, planes.

Situación geomórfica /cuenca fluvial, altitud

Relieve / procesos erosivos

Geología, suelos y sustratos, biología del suelo

Clima (precipitaciones, temperatura, viento)

Régimen hídrico

Áreas de inundación, régimen de flujo, aguas freáticas

Régimen de sedimentos

Calidad del agua

Tipos de hábitat y de humedales

Conectividad

Superficie, límites y dimensiones

Comunidades vegetales, zonas y estructura 1

Comunidades de animales

Especies particulares, raras/amenazadas

Población y actividades socioeconómicas

Ordenación del territorio

Educación, patrimonio cultural, valores estéticos, espirituales y religiosos

Producción primaria $(\mathrm{S})$

Ciclo de nutrientes

Ciclo del carbono

Presiones a la integridad del ecosistema

Productividad de la reproducción animal

Productividad vegetal

Interacciones entre las especies

Dispersión de fauna y flora

Migración

Producción de agua

Tratamiento de aguas y desechos

Alimentos para seres humanos y ganado

Madera, juncos, fibras, turba, productos medicinales

Control de inundaciones

Suelos, sedimentos y retención de nutrientes

Estabilización de costa y riberas

Regulación del clima local

Efecto invernadero

Reabastecimiento de aguas subterráneas

Caza y pesca con fines recreativos

Deportes acuáticos

Actividades de estudio de la naturaleza

Esparcimiento y turismo

Valores educativos, estéticos, espirituales y religiosos

Cultura contemporánea, artes, valores de existencia

Conocimientos e investigaciones

Conflictos en la provisión de servicios ecológicos
1

1

1

1

1

1

1

1

1

1

1

1

1

1

1

1

1

1

18

1

1

1

1

1

1

1

1

1

1

1

1

1

1

1

1

1

1

1

1

1

1

1

1

1

1

1

27

Fuente: elaboración propia a partir de los datos de la Secretaría de la Convención de Ramsar (2010, p. 14). 
Tabla 2. Matriz Multicriterio para Humedales de la Convención de Ramsar (componentes 3, 4 y 5)

\begin{tabular}{|c|c|c|c|}
\hline Componentes $\mathrm{X}$ & Variables $(\mathbf{m})$ & Atributos (j) & $\begin{array}{l}\text { Base Ramsar } \\
\text { peso } W\end{array}$ \\
\hline \multicolumn{4}{|l|}{ 3. Evaluación } \\
\hline Ecológicas & $\begin{array}{l}\text { 16.Hábitats / especies / } \\
\text { procesos hidrológicos } \\
\text { / geológicas y } \\
\text { geomorfológicas / } \\
\text { paisajísticas }\end{array}$ & $\begin{array}{l}\text { Tamaño, balance hídrico, diversidad biológica, naturalidad, } \\
\text { rareza, representatividad, mejoramiento y/o restauración. } \\
\text { Aplicar técnicas de evaluación ambiental estratégica, } \\
\text { impacto ambiental, evaluación de sensibilidad, vulnerabi- } \\
\text { lidad, evaluación rápida de la biodiversidad) / Análisis de } \\
\text { fortalezas y debilidades (Matriz FODA). }\end{array}$ & 1 \\
\hline $\begin{array}{l}\text { Socioeconómicas } \\
\text { y culturales }\end{array}$ & $\begin{array}{l}\text { 17. Bienes y servicios de los } \\
\text { humedales que promueven } \\
\text { el bienestar humano y } \\
\text { rasgos culturales }\end{array}$ & $\begin{array}{l}\text { Estructuras culturales, importancia religiosa y de culto, } \\
\text { investigación científica y monitoreo. Aplicar técnicas } \\
\text { de valoración económica de los humedales, impacto } \\
\text { ambiental, evaluación de riesgos en humedales, análisis de } \\
\text { fortalezas y debilidades (Matriz FODA). }\end{array}$ & 1 \\
\hline \multicolumn{3}{|l|}{ Total valor 3} & 2 \\
\hline \multirow{4}{*}{ 4. Objetivos } & 18. Factores & $\begin{array}{l}\text { Políticas, estrategias, tendencias, limitaciones, } \\
\text { prácticas, conflictos de interés y obligaciones }\end{array}$ & 1 \\
\hline & $\begin{array}{l}\text { 19. Indicadores de } \\
\text { resultados }\end{array}$ & Ecológicos, socioeconómicos y culturales & 1 \\
\hline & 20. Objetivos & $\begin{array}{l}\text { Lineamientos por objetivos específicos para alcanzar la } \\
\text { imagen objetivo }\end{array}$ & 1 \\
\hline & 21. Justificación & $\begin{array}{l}\text { Incentivos, plan de acciones (medidas) para alcanzar la } \\
\text { imagen objetivo }\end{array}$ & 1 \\
\hline \multirow{5}{*}{ 5. Plan de Acción } & \multirow{2}{*}{ 22. Proyectos } & Estrategias & 1 \\
\hline & & Programas y proyectos de manejo & 1 \\
\hline & 23. Programa de trabajo & Cronograma anual de ejecución & 1 \\
\hline & 24. Examen anual & Programa de monitoreo ambiental & 1 \\
\hline & $\begin{array}{l}\text { 25. Examen principal o } \\
\text { auditoría }\end{array}$ & Lineamientos, programa y cronograma de auditoría & 1 \\
\hline \multicolumn{2}{|c|}{ Total valor 4 Y 5} & & 9 \\
\hline \multicolumn{2}{|c|}{ Valor Total Integrado (VTI) } & $\mathrm{V} 1+\mathrm{V} 2.1+\mathrm{V} 2.2+\mathrm{V} 2.3+\mathrm{V} 3+\mathrm{V} 4+\mathrm{V} 5$ & 56 \\
\hline
\end{tabular}

Fuente: elaboración propia a partir de los datos de la Secretaría de la Convención de Ramsar (2010, p. 14).

Los valores $x$ mij son asignados en función a las variables y atributos que contiene las PEMHs de cada país según el MHCR. La valoración consiste en asignar el valor de $(0 ; 0,20 ; 0,40 ; 0,60 ; 0,80 \mathrm{y} / \mathrm{o}$ $1,00)$ para el $i$ modelo país que incluye el atributo $j$ de Ramsar del criterio $m$ y componente $x$.

La sumatoria de los xmji atributos da como resultado el valor de los 5 componentes para un total igual a 56, ( $x$ mji $=56)$. El resultado es el Valor Total Integrado, (VTI) igual a 56 para Ramsar, el cual se relaciona con el Índice de Modelo de Gestión de Humedales (IMGH) (Ecuación 1).

$$
\boldsymbol{I M G H}=\frac{\sum \boldsymbol{w} * \boldsymbol{m} \boldsymbol{j} \boldsymbol{i}}{\sum \boldsymbol{j}} \leq \mathbf{1} \quad \text { Ecuación } 1
$$

La relación de la sumatoria de [ $\left.\mathrm{w}^{*} \mathrm{mji}\right]$ con respecto a la sumatoria de atributos $[\mathrm{Xm}] \mathrm{j}$ de Ramsar, se clasifica en un rango de categorías desde muy bajo entre $(0,09-0,29)$, bajo entre $(0,30-0,49)$, mediano entre $(0,50-0,69)$, alto entre $(0,70-0,89)$ y muy alto valor entre $(0,90-1,00)$. 


\section{Formulación del modelo para la gestión del Humedal Urama (HU)}

El componente técnico del modelo para la gestión del HU está asociado a la conformación de una estructura que describe los componentes y variables de gestión, cuyo dominio categoriza y ordena atributos, considerando los resultados obtenidos del análisis de las PEMHs, donde para el componente evaluación se plantea incluir un eje transversal que consiste en las variables de impacto como estrategia para la restauración, estructura que puede contribuir a delimitar las unidades de ordenamiento del humedal para la elaboración y posterior implementación del Plan de Acción y su seguimiento.

La formulación del modelo para la gestión del HU queda definida por la sumatoria de los componentes que integran la estructura para el cálculo de IMGH integral (ecuación 2), siendo: IMGH_P (Política); IMGH_CE (Características Ecológicas); IMGH_PE (Procesos Ecológicos); IMGH_SE (Servicios de Ecosistemas) + IMGH_E (Evaluación) +IMGH_O (Objetivos) +IMGH_PAc (Plan de Acción).

$$
\begin{gathered}
\text { IMGH = IMGH_P + IMGH_CE+ IMGH_PE+ IMGH_ } \\
\text { SE + IMGH_E+IMGH_O+IMGH_PAc. }
\end{gathered}
$$

Ecuación 2

\section{Resultados}

\section{Resultado de la MMC de gestión de humedales}

Como resultado de la MMC para el modelo de gestión se obtienen los siguientes: Argentina: 1) Política (P) y características ecológicas (CE): 16; 2) Procesos (PE) y servicios de ecosistemas, (SE):14; 3) Evaluación: 1,4; 4) Objetivos (O) y Plan de Acción (PAc): 5; VTI=36,4; IMGH= 0,65; categoría mediano. En adelante se resume como sigue: Brasil
1) 9,4 ; 2) 9,6 ; 3) 2 ; 4) 7; VTI= 28, IMGH=0,50; categoría bajo; Bolivia: 1) 8,8; 2) 11,8;3) 1; 4) 5; $\mathrm{VTI}=26,6$; IMGH=0,48; categoría bajo; Chile, 1) 17; 2) 23;3) 2; 4) 9; VTI= 49 IMGH=88; categoría alto. Colombia 2 1) 12; 2) 16; 3) 2; 4) 9; VTI= 39; IMGH $=0,73$; categoría mediano. Costa Rica, 1$)$ 16,4; 2) 18; 3) 2, 4) 9; VTI=46,4; IMGH=0,81; categoría alto. España, 1) 17; 2) 23; 3) 2; 4) 9; VTI=51; IMGH=0,91; categoría muy alto; Guatemala 1) 11; 2) 161 ; 3) 1,4 ; 4) 6; VTI= 34,4; IMGH=0,61; categoría alto; Honduras 1) 18; 2) 16; 3) 2; 4) 7; VTI= 43; IMGH=0,77; categoría alto; México, 1) 18; 2) 23; 3) 2; 4) 8; VTI=51, IMGH=0,91, categoría muy alto; Nicaragua, 1) 10,2; 2) 11,6;3) 1,6;4) 4; VTI=27,4; IMGH=0,49; categoría bajo; Panamá, 1) 16,6 ; 2) 15,8 ; 3) 2; 4) 8; VTI=42,4; IMGH=0,76; categoría alto; Paraguay: 1)11;2) 6; 3) 1,2; 4) 8; VTI = 26,2; $\mathrm{IMGH}=0,47$; categoría bajo; Perú, 1) 13 ; 2) 17,2 ; 3) 1 ,2; 4) 8 , VTI= 39,4, IMG $=0,70$, categoría mediano. Venezuela: 1) 14; 2) 11;3) 0,8; 4) 2, VTI=27,8; IMGH=0,50; categoría bajo.

En la Tabla 3 se presenta una muestra de los resultados correspondientes a la MMC del IMGH para 7 países: Argentina, Brasil, Bolivia, Chile, Colombia, Costa Rica y España.

El IMGH obtenido según el rango es el siguiente: Categoría muy alto: España, México (13,33\%); categoría alto: Chile, Colombia, Costa Rica, Honduras, Panamá, (33,33\%); categoría mediana: Argentina, Guatemala, Perú, (20\%); categoría bajo: Brasil, Bolivia, Nicaragua, Paraguay, Venezuela $(33,33 \%)$. No se obtienen resultados con la categoría muy bajo. Se observa una tendencia al incremento desde la categoría baja a muy alta hacia el valor de Ramsar, (IMGH=1), representado por la ecuación de línea recta $Y=0,037 X+0,3784$; donde 10 países se encuentran desde la categoría "mediano a muy alto" para un $66,67 \%$ y un $33,33 \%$ en la categoría baja (Figura 2). 
Tabla 3. Resultado de MMC de IMGH para países de AM y España

\begin{tabular}{|c|c|c|c|c|c|c|c|c|c|}
\hline \multirow[b]{2}{*}{$\begin{array}{c}\text { Componentes } \\
\text { (X) }\end{array}$} & \multirow[b]{2}{*}{$\begin{array}{l}\text { Variables } \\
\quad(\mathbf{m})\end{array}$} & \multicolumn{8}{|c|}{$\mathbf{N}^{\circ}$ de atributos (i) } \\
\hline & & Ramsar & $\begin{array}{c}1 . \\
\text { Argentina }\end{array}$ & $\begin{array}{c}2 . \\
\text { Brasil }\end{array}$ & $\begin{array}{c}3 . \\
\text { Bolivia }\end{array}$ & $\begin{array}{c}4 . \\
\text { Chile }\end{array}$ & $\begin{array}{c}5 . \\
\text { Colombia }\end{array}$ & $\begin{array}{c}6 . \\
\text { Costa Rica }\end{array}$ & $\begin{array}{c}7 . \\
\text { España }\end{array}$ \\
\hline 1. Política & $\begin{array}{l}\text { Política y estrategias de } \\
\text { humedales y / o planes } \\
\text { relacionados }\end{array}$ & 1 & 1 & 1 & 1 & 1 & 1 & 1 & 1 \\
\hline $\begin{array}{l}\text { 2. Descripción } \\
\text { / características } \\
\text { ecológicas }\end{array}$ & $\begin{array}{l}\text { 2.1 Componentes } \\
\text { ecológicas, socio- } \\
\text { económicos y culturales }\end{array}$ & 17 & 15 & 8,4 & 7,8 & 16 & 11 & 9 & 16 \\
\hline Subtotal valor 1 & & 18 & 16 & 9,4 & 8,8 & 17 & 12 & 16,4 & 17 \\
\hline 2. Descripción & 2.2 Procesos ecológicos & 9 & 4 & 2 & 4,2 & 9 & 3 & 2 & 8 \\
\hline $\begin{array}{l}\text { / características } \\
\text { ecológicas }\end{array}$ & $\begin{array}{l}\text { 2.3 Servicios de } \\
\text { ecosistemas }\end{array}$ & 18 & 10 & 7,6 & 7,6 & 14 & 15 & 16 & 15 \\
\hline Subtotal valor 2 & & 27 & 14 & 9,6 & 11,8 & 23 & 18 & 18 & 23 \\
\hline 3. Evaluación & $\begin{array}{l}\text { Ecológicos, socio- } \\
\text { económicos y culturales }\end{array}$ & 2 & 1,4 & 2 & 1 & 2 & 2 & 2 & 2 \\
\hline Total valor 3 & & 2 & 1,4 & 2 & 1 & 2 & 2 & 2 & 2 \\
\hline 4. Objetivos & $\begin{array}{l}\text { Factores, indicadores, } \\
\text { objetivos, motivación }\end{array}$ & 4 & 2 & 4 & 3 & 4 & 4 & 4 & 4 \\
\hline 5. Plan de acción & $\begin{array}{l}\text { Proyectos, programa, } \\
\text { examen anual, auditoría }\end{array}$ & 5 & 3 & 3 & 2 & 5 & 5 & 5 & 5 \\
\hline Total valor 4 & & 9 & 5 & 7 & 5 & 9 & 9 & 9 & 9 \\
\hline $\begin{array}{l}\text { Valor Total } \\
\text { Integrado (VTI) }\end{array}$ & & 56 & 36,4 & 28 & 26,6 & 49 & 41 & 45,4 & 51 \\
\hline IMGH & & 1 & 0,65 & 0,50 & 0,48 & 0,88 & 0,73 & 0,81 & 0,91 \\
\hline
\end{tabular}

Fuente: elaboración propia según resultado de MMC de IMGH para países de AL y España.

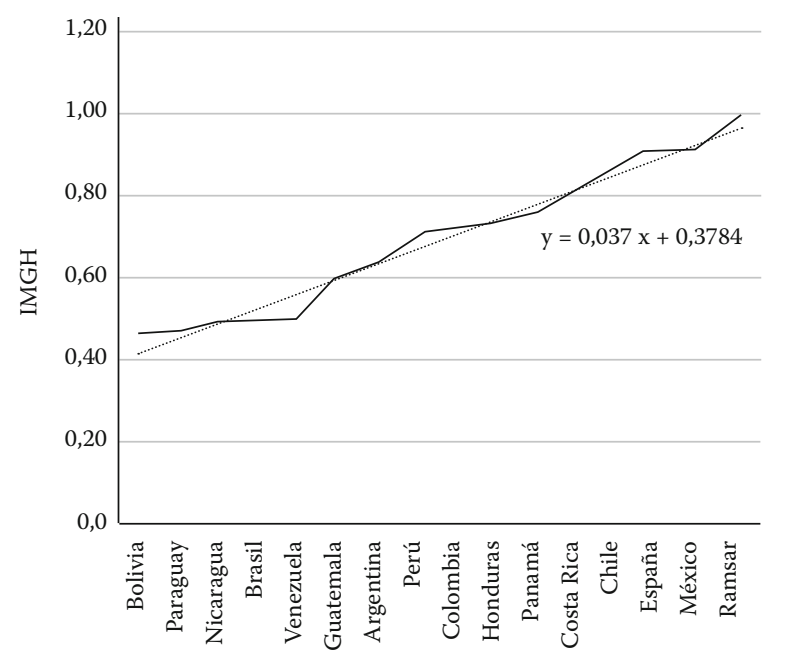

Figura 2. Tendencia del Î́ndice del Modelo de Gestión para Humedales (IMGH). Fuente: elaboración propia según resultado de MMC de IMGH para países de AL y España.

\section{Análisis del resultado obtenido}

Los valores del IMGH clasificados según las componentes y expresados en términos de porcentajes se indican en la Tabla 4, presentando una muestra para 8 países: 1 . Argentina; 2 . Brasil; 3. Bolivia; 4. Chile; 5. Colombia; 6. Costa Rica; 7. España y 8. Venezuela.

Del resultado se analiza lo siguiente:

1. Política (P): El atributo se presenta $100 \%$ en los países del estudio, con excepción de Venezuela (60\%), debido que a la fecha no cuenta con una PEMH. La PEMH está basada en los lineamientos establecidos por la Convención de Ramsar, dando cumplimiento a las resoluciones de los planes estratégicos, se clasifica en 4 planes estratégicos de Ramsar (PER). Las PEMHs vinculadas son: 1. PER 
(1997-2002): 1 país (España); 2.PER (2003-2008): 2 países (Colombia y Nicaragua); 3. PER (20092015): 4 países (Argentina, México, Paraguay y Perú); 4. PER (2016-2024): 7 países (Brasil, Bolivia, Chile, Guatemala, Honduras y Panamá).

2. Descripción de características ecológicas (DCE): Comprende el inventario del humedal (IH), clasificado en tres variables: características ecológicas $(\mathrm{CE})$, procesos ecológicos $(\mathrm{PE})$ y servicios del ecosistema (SE), dando cumplimiento a la estrategia 2.4 del PER (2009-2015) que consiste en mantener las características ecológicas de todos los humedales mediante actividades de planificación y manejo (Secretaría de la Convención de Ramsar, 2010, p. 5 ). El componente DCE se ha incluido con el objetivo específico de la elaboración o actualización del IH, indicando las acciones del manejo, siendo: actualización, delimitación, ordenamiento territorial y la frecuencia temporal (Ministerio de Medio Ambiente, España, 2001, p. 55; Ministerio de Ambiente y Energía de Costa Rica, 2017, p. 42; Ministerio de Ambiente de Panamá, 2018, p. 39).

Los resultados para las CE son: 1 . categoría muy alto: España, México y Chile con 94,12\% cada uno, Panamá $(91,76 \%)$ y Costa Rica $(90,59 \%) ; 2$. categoría alto: Argentina 88,24\%, Honduras (82,35\%), Venezuela $(78,82 \%)$, Perú $(70,59 \%) ; 3$. categoría mediana: Colombia 64,71\%, Guatemala (58,82\%), Paraguay $(58,82 \%)$, Nicaragua $(54,12 \%)$; 4 . categoría bajo: Brasil $(49,41 \%)$ y Bolivia $(45,88 \%)$.
Para las variables de PE y SE resulta: 1. categoría muy alto: no se obtienen resultados; 2 . categoría alto: México (88,89), España y Chile con $85,19 \%$ cada uno; 3. Categoría mediano: Colombia y Costa Rica con $66,67 \%$ cada uno, Perú $(63,70 \%)$, Guatemala y Honduras con 59,26\% cada uno, Panamá (58,52\%), Argentina (51,85\%); 4. Categoría bajo: Bolivia (43,70\%); Nicaragua (42,96\%), Venezuela $(40,74 \%)$ y Brasil (35,56\%). No se presentan resultados con categoría muy bajo. Como muestra, la PEMH de México presenta la relación de atributos de PE y SE como control de inundaciones, depuración del agua (SEMARNAT, 2010, p. 50).

3. Evaluación (E): 1. Categoría muy alto: 100\% en ocho (8) países (Brasil, Chile, Colombia, Costa Rica, España, Honduras, México y Panamá); 2. Categoría alto: Nicaragua (80\%); 3. Categoría mediano: Argentina y Guatemala con $70 \%$ cada uno, Paraguay y Perú con 60\%; 4. Categoría bajo: Bolivia (50\%) y Venezuela (40\%). El promedio de este componente es un $82 \%$. Este componente comprende los atributos para el cumplimiento de los objetivos y metas establecidos de la PER, donde se incluye evaluar la restauración de humedales, el cambio climático, promover las evaluaciones ecológicas y valoraciones económicas de los beneficios y funciones de los humedales (República de Colombia. Ministerio del Medio Ambiente, 2002, p. 40); la PEMH de Costa Rica incluye mapear y determinar actividades productivas congruentes

Tabla 4. Valor del IMGH por componentes expresado en porcentajes (\%).1. Argentina; 2. Brasil; 3. Bolivia; 4. Chile; 5. Colombia; 6. Costa Rica; 7. España y 8. Venezuela

\begin{tabular}{|c|c|c|c|c|c|c|c|c|c|c|}
\hline \multirow{2}{*}{ Componentes } & \multirow{2}{*}{ Ramsar } & \multicolumn{8}{|c|}{ Valor del IMGH en porcentajes (\%) } & \multirow{2}{*}{$\begin{array}{c}\text { Promedio } \\
\%\end{array}$} \\
\hline & & 1. & 2. & 3. & 4. & 5. & 6. & 7. & 8. & \\
\hline 1. Política & 100,00 & 100,00 & 100,00 & 100,00 & 100,00 & 100,00 & 100,00 & 100,00 & 60,00 & 97,33 \\
\hline $\begin{array}{l}\text { 2.1. Caracterísitcas } \\
\text { ecológicas-componentes }\end{array}$ & 100,00 & 88,24 & 49,41 & 45,88 & 94,12 & 64,71 & 90,59 & 94,12 & 78,82 & 74,43 \\
\hline $\begin{array}{l}\text { 2.2. Procesos ecológicos } \\
\text { 2.3. Servicios de ecosistemas }\end{array}$ & 100,00 & 51,85 & 35,56 & 43,70 & 85,19 & 66,67 & 66,67 & 85,19 & 40,74 & 58,02 \\
\hline 3. Evaluación & 100,00 & 70,00 & 100,00 & 50,00 & 100,00 & 100,00 & 100,00 & 100,00 & 40,00 & 82,00 \\
\hline $\begin{array}{l}\text { 4. Objetivos y } \\
\text { 5. Plan de Acción }\end{array}$ & 100,00 & 55,56 & 77,78 & 55,56 & 100,00 & 100,00 & 100,00 & 100,00 & 22,22 & 77,04 \\
\hline VTI & 100,0 & 65,0 & 50,0 & 47,5 & 87,5 & 73,2 & 81,1 & 91,1 & 49,6 & 67,4 \\
\hline
\end{tabular}

Fuente: elaboración propia según resultado de MMC de IMGH para países de AL y España. 
con los usos sostenibles del humedal (Ministerio de Ambiente y Energía de Costa Rica, 2017, p. 47).

4. Objetivos y Plan de acción (PAc): 1. Categoría muy alto: $100 \%$ es obtenido para Chile, Colombia, Costa Rica y España; 2. Categoría alto: 88,89\% es obtenido para México, Panamá, Paraguay y Perú; 77,78\% para Brasil y Honduras; 3. Categoría mediano: Guatemala (66,67\%), Argentina y Bolivia con 55,56\%. 4. Categoría bajo: no se obtienen resultados; 5 . Categoría muy bajo: Venezuela (22\%). Este componente es una estrategia que incluye el cumplimiento de los objetivos y metas del PER vinculados con los Objetivos de Desarrollo Sostenible por los países de AL y España. Los resultados indican el cumplimiento por parte de 14 países de AL y España; donde para el vigente PER (2016-2024) (Convención de Ramsar, 2015, p. 10), 7 países han elaborado la PEMH, manteniendo variantes entre sus atributos. Se destaca la categoría muy baja de Venezuela, por cuanto no cumple con este lineamiento de la Convención de Ramsar, por lo cual, el Plan de Acción está basado sólo en el marco legal venezolano donde se establece la elaboración de los planes de manejo y reglamento de uso para las AP (República de Venezuela, 1983, p. 11), siendo requerida la vinculación con la PER (2016-2024).
Entre las PEMHs, el componente con mayor variación es el segundo que corresponde a la DCE, el cual es clasificado a su vez en CE con $74,43 \%$ y PE-SE con $58,02 \%$. En el siguiente orden se encuentran los objetivos y PAc alcanzan el 77,04\%; siguiendo el tercer componente Evaluación con $82 \%$ y la política alcanza el $97,33 \%$, debido al resultado de Venezuela. Como valor integrado, los modelos de gestión incluyen los componentes para la planificación en un $67,4 \%$ con respecto a MHCR (Tabla 4). En la Tabla 5 para el análisis de la PEMH se presenta la relación del IMGH por componentes y variables, incluye el resultado del $\mathrm{IMGH}_{\text {integral }}=0,67$.

Comparando los resultados con COMTEMAOLACEFS (2015, p. 15), para la evaluación del cumplimento por parte de los gobiernos nacionales del acuerdo establecido por la Meta 11 de Aichi en el marco de la Convención de las Naciones Unidas sobre la Diversidad Biológica, la herramienta utiliza el Indicador de Implementación de Gestión (IIG) de las AP para 1120 áreas evaluadas en América Latina para tres categorías. Se obtiene que $19 \%$ se encuentra en la categoría alto, $52 \%$ en la categoría medio y $29 \%$ en categoría bajo.

Tabla 5. Relación del IMGH integral por componentes de la MHCR

\begin{tabular}{|c|c|c|c|c|c|}
\hline Componentes (X) & Variables $(\mathbf{m})$ & $\begin{array}{l}\mathbf{N}^{\circ} \text { Atributos } \\
\text { RAMSAR (j) }\end{array}$ & $\begin{array}{c}\text { Total } \\
\text { atributos }\end{array}$ & Promedio & $\begin{array}{l}\text { IMGH por } \\
\text { componente }\end{array}$ \\
\hline 1. Política & $\begin{array}{l}\text { Política y estrategias de humedales } \\
\text { y / o planes relacionados }\end{array}$ & 1 & 14,6 & 0,97 & 0,97 \\
\hline $\begin{array}{l}\text { 2. Descripción / características } \\
\text { ecológicas }\end{array}$ & $\begin{array}{l}\text { 2.1. Componentes ecológicas, socioeconómicos } \\
\text { y culturales }\end{array}$ & 17 & 189,8 & 12,65 & 0,74 \\
\hline Subtotal valor 1 & & 18 & 204,4 & 13,63 & 0,76 \\
\hline \multirow{2}{*}{$\begin{array}{l}\text { 2. Descripción / características } \\
\text { ecológicas }\end{array}$} & 2.2. Procesos ecológicos & 9 & 59,8 & 3,99 & 0,44 \\
\hline & 2.3. Servicios de ecosistemas & 18 & 175,2 & 11,68 & 0,65 \\
\hline Subtotal valor 2 & & 27 & 235 & 15,67 & 0,58 \\
\hline 3. Evaluación & Ecológicos, socioeconómicos y culturales & 2 & 24,6 & 1,64 & 0,82 \\
\hline Total valor 3 & & 2 & 24,6 & 1,64 & 0,82 \\
\hline 4. Objetivos & Factores, indicadores, objetivos, motivación & 4 & 51,2 & 3,41 & 0,85 \\
\hline 5. Plan de acción & Proyectos, programa, examen anual, auditoría & 5 & 52,8 & 3,52 & 0,70 \\
\hline Total valor 4 & & 9 & 104 & 6,93 & 0,77 \\
\hline Valor Total Integrado (VTI) & & & 566 & 37,73 & 0,67 \\
\hline $\begin{array}{l}\text { Índice de Modelo de Gestión de } \\
\text { Humedales (IMGH)integral }\end{array}$ & & & & 0,67 & \\
\hline
\end{tabular}

Fuente: elaboración propia según resultado de MMC de IMGH para países de AL y España. 
La integración del IMGH para las categorías muy alto, alto y mediano, arrojan un total de $66,67 \%$ muy por encima del promedio y un 33,33\% para la categoría bajo. Para los porcentajes del indicador de implementación de gestión, (IIG), la categoría alto y la categoría medio alcanzan un porcentaje de $71 \%$ por encima del promedio y la categoría bajo presenta una relación de $29 \%$, por lo que se mantiene una relación por encima del promedio de la gestión.

Según COMTEMA-OLACEFS (2015, p. 15), con relación a valores particulares de implementación de gestión por país y categorías (alto, medio y bajo) se obtiene: Costa Rica (16, 40 y 44) \%, México (35, 43 y 22$) \%$, Venezuela, (25, 50 y 25) \%, Argentina, $(39,61$ y 0$) \%$, Colombia $(22,69$ y 9) \%. En la Tabla 6 se muestras resultados comparativos de la investigación con el estudio de COMTEMA-OLACEFS, (2015, p. 15).

La comparación de los resultados de la investigación muestra correspondencia con 4 valores de implementación de la gestión según COMTEMAOLACEFS (2015, p. 15). Para México y Colombia se mantienen en una categoría de muy alto a alto; para Costa Rica y Argentina en una categoría de mediano a alto; mientras que para Venezuela el valor de la planificación (IMGH) tiene una categoría baja considerando la debilidad de no cumplir con una PEMH; sin embargo, para la implementación se obtuvo una categoría alta en el año 2015, lo cual puede atribuirse a las estrategias de las AP con un
$50 \%$ de los planes de manejo, mediante el rediseño de la metodología para la elaboración de Planes de Ordenación y Reglamentos de Uso (INPARQUES, 2012, p. 43).

\section{Desarrollo del Modelo para la Gestión del HU}

El modelo para la gestión del HU considera los resultados obtenidos de la MMC para el IMGH donde las categorías muy alto y alto clasifica a los modelos de España, México, Chile, Colombia, Costa Rica, Honduras y Panamá, caracterizados por considerar factores claves de los PER.

La aplicabilidad del modelo consiste en la definición del área de protección del humedal dentro de una unidad de estudio que es la cuenca hidrográfica del río Urama, planteando un proceso con enfoque ecosistémico integral sobre los componentes de gestión atendiendo la variación de los modelos analizados como es: CE, PE-SE seguidos del PAc, Evaluación y Política. La formulación según los resultados de la Tabla 5 y la Ecuación 2 es la siguiente:

$$
\begin{aligned}
& \mathrm{IMGH}_{\text {integral }}=0,97\left(\mathrm{IMGH} \_\mathrm{P}\right)+0,74\left(\mathrm{IMGH} \_\mathrm{CE}\right) \\
& \text { + 0,44(IMGH_PE) + 0,65(IMGH_SE) } \\
& +0,82(\text { IMGH_E })+0,85(\text { IMGH_O })+ \\
& \text { 0,70(IMGH_PA). }
\end{aligned}
$$

\begin{tabular}{|c|c|c|c|c|c|c|c|}
\hline \multirow[t]{2}{*}{ Pais } & \multicolumn{2}{|c|}{$\begin{array}{l}\text { Índice de Modelo } \\
\text { de Gestión de Humedales, (IMGH) (\%) } \\
\text { (Planificación) INVESTIGACIÓN, } 2020\end{array}$} & \multicolumn{5}{|c|}{$\begin{array}{l}\text { Indicador de Implementación de Gestión, (IIG) (\%) } \\
\text { COMTEMA - OLACEFS, } 2015\end{array}$} \\
\hline & $\%$ & Categoría & Alto & Medio & Sub. Total & Bajo & Categoría \\
\hline Costa Rica & 81,07 & Alto & 16 & 40 & 56 & 44 & Mediano \\
\hline México & 91,07 & Muy alto & 35 & 43 & 78 & 22 & Alto \\
\hline Venezuela & 49,64 & Bajo & 25 & 50 & 75 & 25 & Alto \\
\hline Argentina & 65,00 & Mediano & 39 & 61 & 100 & 0 & Muy alto \\
\hline Colombia & 73,21 & Alto & 22 & 69 & 91 & 9 & Muy alto \\
\hline
\end{tabular}

Lo novedoso del modelo para la gestión del HU es la aplicación de las variables de impacto en el tercer

Tabla 6. Índice de Modelo de Gestión Vs. Indicador de Implementación de Gestión

Fuente: elaboración propia según datos de la MMC de IMGH para países de AL y España y COMTEMA-OLACEFS (2015, p. 15). 
componente (Evaluación) como un eje transversal a la estructura; es decir, dinámica de cambios UTCT, hidrografía (balance hídrico), sensibilidad ambiental y valoración ambiental, siendo una estrategia de restauración para el control del cambio de las características ecológicas en función de las posibles causas-efectos generados por los sectores claves que se desarrollan o puedan desarrollarse en el área del humedal. En el diagrama de la Figura 3 se muestran los componentes del modelo propuesto.

Modelo para la gestión del humedal Urama

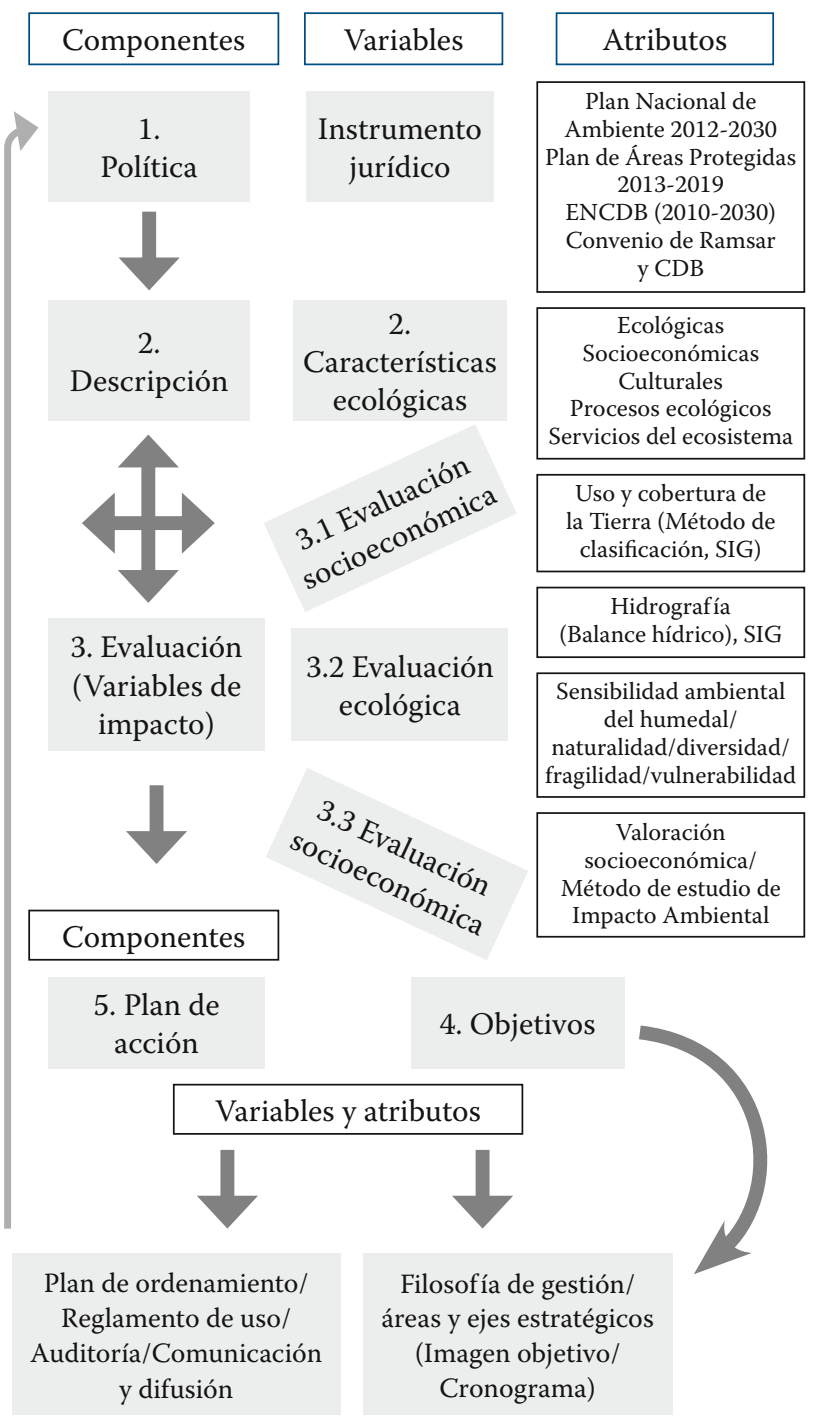

Figura 3: Diagrama sinóptico del modelo para la gestión del humedal Urama. Fuente: elaboración propia a partir de datos de la Secretaría de la Convención de Ramsar (2010, p. 14).
El diseño de modelo de gestión está compuesto por 5 componentes, 28 variables y 56 atributos, para el proceso de inventario, evaluación y el plan de acción (tablas 7 y 8).

La descripción de las CE, PE y SE comprende el IH a partir de los atributos de MHCR. Para la evaluación ecológica y socioeconómica a ser desarrollada, consiste en cuatro atributos:

1) Dinámica de UTCT, factores determinantes para la restauración y ordenamiento territorial del área de humedal, considerando los posibles cambios de las características ecológicas. Se aplica el método de clasificación en plataformas de SIG, con los programas ERDAS, ENVI y ArcGIS y las imágenes del satélite LANDSAT (Márquez, Guevara y Rey, 2018a, p. 3).

2) Balance hídrico de la cuenca para un período de tiempo aplicando el método geoestadístico espacio-temporal, para la proyección y el pronóstico de las variables hidrológicas (Márquez, Guevara y Rey, 2018b, p. 3); aporta resultados para la definición del ordenamiento territorial que se integra junto con los resultados de la dinámica de UTCT.

3) Estudio de sensibilidad ambiental ante las acciones de proyectos de desarrollo que puedan darse en el área del humedal (PEQUIVEN, 2014 , p. 8; Rojas, 1985 , p. 29), se determinan las áreas sensibles y los efectos ambientales, estudio relevante para los proyectos de restauración y el ordenamiento territorial.

4) Valoración económica de los servicios del ecosistema, aplicando el método de Estudio de Impacto Ambiental (De Groot et al., 2007, p. 22), información a ser considerada para formular los objetivos y el correspondiente PAc.

Los objetivos y el PAc se basan en los propósitos estratégicos establecidos en el PER (2016-2024), comprendidos por 19 metas respecto a la integración de políticas y estrategias nacionales, uso del agua, funciones y servicios, planes de manejo, programas y proyectos a escala de cuenca hidrográfica para restauración de las $\mathrm{CE}$, control de 
especies invasoras, control de impactos, sostenibilidad de sectores claves, actualización de $\mathrm{IH}$, seguimiento y monitoreo; conocimiento tradicional y las prácticas de los pueblos indígenas, investigación científica, coordinación institu- cional, cooperación financiera (Convención de Ramsar, 2015, p. 14); se incluyen atributos del ordenamiento legal vigente en Venezuela para AP (República de Venezuela, 1983, p. 11) vinculado con la PER (2016-2024).

Tabla 7. Matriz integrada del modelo para la gestión del humedal Urama (componentes 1 y 2 )

\begin{tabular}{|c|c|c|}
\hline Componentes & Variables & Atributos \\
\hline 1. Política & 1. Instrumento jurídico & $\begin{array}{l}\text { Plan Nacional de Ambiente 2012-2030 } \\
\text { Plan Estratégico de Áreas Protegidas 2013-2019 } \\
\text { Estrategia Nacional 2010-2020 y su plan de acción. } \\
\text { PER (2016-2024) }\end{array}$ \\
\hline \multirow{3}{*}{$\begin{array}{l}\text { 2. Descripción / características } \\
\text { ecológicas (Secretaría de la Convención } \\
\text { de Ramsar, 2010) }\end{array}$} & 2. Geología & Geología, sismicidad. \\
\hline & 3. Geomorfología & Paisaje/ procesos morfodinámicos / unidades \\
\hline & 4. Suelo & Unidades de suelos, perfil del suelo, nivel freático \\
\hline \multirow{15}{*}{$\begin{array}{l}\text { 2.1. Componentes ecológicos, } \\
\text { socioeconómicos y culturales }\end{array}$} & 5. Clima & Variables climáticas /clasificación \\
\hline & 6. Hidrografía & Estudio hidrológico e hidráulico \\
\hline & & Áreas de inundación \\
\hline & & Calidad del agua \\
\hline & 7. Atmósfera & Calidad del aire \\
\hline & 8. Ecosistemas & Tipos de hábitats, humedal \\
\hline & & Conectividad de los hábitats \\
\hline & & Superficie, límites y dimensiones \\
\hline & 9. Vegetación & Comunidades, zonas y estructura \\
\hline & 10. Fauna & Fauna silvestre \\
\hline & & Especies particulares, raras, amenazadas e invasoras \\
\hline & 11. Socioeconómicos & Población \\
\hline & & Ordenación del territorio \\
\hline & & Equipamiento e infraestructura de servicios \\
\hline & 12. Culturales & Organización comunitaria \\
\hline \multirow[t]{3}{*}{ 2.2. Procesos ecológicos } & 13. Ecosistemas & Funcionalidad \\
\hline & & Vulnerabilidades a la integridad del ecosistema \\
\hline & $\begin{array}{l}\text { 14. Vegetación } \\
\text { 15. Fauna }\end{array}$ & Especies según inventario en sitio \\
\hline \multirow[t]{12}{*}{ 2.3. Servicios de ecosistemas } & 16. Aprovisionamiento & Producción de agua \\
\hline & & Tratamiento de aguas / residuos y desechos \\
\hline & & Alimentos para personas \\
\hline & 17. Regulación & Regulación de inundaciones \\
\hline & & Regulación del clima \\
\hline & & Regulación biológica \\
\hline & 18. Socioeconómico & Condiciones ocupacionales de la población \\
\hline & & Sistemas de ciudades y estructura urbana. \\
\hline & & Uso del suelo y tenencia de la tierra \\
\hline & & Uso turístico y recreacional \\
\hline & 19. Culturales & Valores paisajísticos, estéticos y culturales. \\
\hline & & Sistemas de conocimientos \\
\hline
\end{tabular}

Fuente: elaboración propia a partir de los datos de la Secretaría de la Convención de Ramsar (2010, p. 14). 
Tabla 8: Matriz integrada del modelo para la gestión del humedal Urama (componentes 3,4 y 5 )

\begin{tabular}{|c|c|c|}
\hline Componentes & Variables & Atributos \\
\hline \multirow[t]{4}{*}{ 3. Evaluación } & 20. Uso y cobertura de la tierra & $\begin{array}{l}\text { Detección de cambios UTCT: vegetación, agropecuario, rural, suelo } \\
\text { desnudo, cuerpo de agua. Método de clasificación SIG (ERDAS, } \\
\text { ENVI y ArcGIS) e imágenes del satélite LANDSAT (Márquez, } \\
\text { Guevara y Rey, 2018a, p. 3). }\end{array}$ \\
\hline & 21. Hidrografía & $\begin{array}{l}\text { Modelación de variables de balance hídrico, Método geoestadístico } \\
\text { espacio-temporal (Márquez, Guevara y Rey, 2018b, p. 3). }\end{array}$ \\
\hline & $\begin{array}{l}\text { 22. Sensibilidad ambiental: } \\
\text { Geología / Geomorfología / } \\
\text { Suelos / vegetación / fauna / } \\
\text { socioeconómica y cultural }\end{array}$ & $\begin{array}{l}\text { Tamaño, diversidad biológica, naturalidad, rareza, representati- } \\
\text { vidad: Método de sensibilidad ambiental del humedal (Pequiven, } \\
\text { 2014, p. } 8 \text {; Rojas, 1985, p. 29). }\end{array}$ \\
\hline & $\begin{array}{l}\text { 23. Valoración Socioeconómica } \\
\text { y cultural, bienes y servicios }\end{array}$ & $\begin{array}{l}\text { Servicios de aprovisionamiento, regulación, apoyo y culturales: valo- } \\
\text { ración ambiental según Método de Estudio de Impacto Ambiental } \\
\text { (De Groot, Stuip, Finlayson y Davidson, 2007, p. 22). }\end{array}$ \\
\hline \multirow[t]{5}{*}{$\begin{array}{l}\text { 4. Objetivos } \\
\text { (INPARQUES, 2012) }\end{array}$} & \multirow[t]{5}{*}{ 24. Objetivos } & $\begin{array}{l}\text { Creación de área protegida, exposición de motivos, filosofía de } \\
\text { gestión }\end{array}$ \\
\hline & & Objetivos: general, específicos, visión, misión , principios, criterios \\
\hline & & Áreas y ejes estratégicos \\
\hline & & Imagen Objetivo, escenarios a corto, mediano y largo plazo \\
\hline & & Cronogramas / horizonte de tiempo/ escenarios \\
\hline \multirow[t]{13}{*}{$\begin{array}{l}\text { 5. Plan de Acción } \\
\text { (INPARQUES, 2012) }\end{array}$} & 25. Plan de ordenamiento & $\begin{array}{l}\text { Objeto, exposición de motivos, disposiciones generales, directrices, } \\
\text { lineamientos }\end{array}$ \\
\hline & Unidades de ordenamiento (UO) & Zonas de ordenamiento \\
\hline & \multirow[t]{2}{*}{ Programas y subprogramas } & Programa y subprogramas de gestión \\
\hline & & Comisión técnica e instituciones responsables \\
\hline & \multirow[t]{2}{*}{ Programa de trabajo } & Cronogramas de ejecución \\
\hline & & Monitoreo y seguimiento ambiental \\
\hline & Base económica del plan & Plan de inversión financiero / actividades \\
\hline & \multirow[t]{3}{*}{ 26. Reglamento de uso } & Administración del plan \\
\hline & & Autorizaciones y aprobaciones administrativas \\
\hline & & Asignación de usos y actividades en las UO \\
\hline & \multirow[t]{2}{*}{ 27. Auditoría } & Previsión, control posterior y régimen de protección del humedal \\
\hline & & Programa de guardería ambiental y cronograma de auditoría \\
\hline & 28. Comunicación y difusión & $\begin{array}{l}\text { Disposiciones transitorias y finales /Instituciones / organizaciones / } \\
\text { sociedad comunal }\end{array}$ \\
\hline
\end{tabular}

Fuente: Elaboración propia a partir de datos de la Secretaría de la Convención de Ramsar (2010, p. 14).

\section{Conclusiones}

Los resultados del análisis de las PEMHs muestran una tendencia del IMGH al incremento para alcanzar el valor total de los atributos de la MHCR, para lo cual un 13,33\% corresponde a la categoría muy alta (España y México), un 33,33\% alcanza la categoría alta (Chile, Colombia, Costa Rica,
Honduras, Panamá), un 20\% alcanza la categoría mediana (Argentina, Guatemala, Perú), y un 33,33\% corresponde a la categoría baja, (Brasil, Bolivia, Nicaragua, Paraguay, Venezuela), obteniendo el promedio de la gestión por encima del $67 \%$.

Para los modelos analizados, el componente que presenta mayor variación es la Descripción de 
Características Ecológicas clasificado en 78,43\% para CE y $58,02 \%$ para PE y SE, respectivamente; seguido de los objetivos y PAc con 77\%; en el siguiente orden se clasifica a la Evaluación con $82 \%$. La Política alcanza el $100 \%$ en 14 países y un $60 \%$ para Venezuela, al no contar con una PEMH. Esta variación se atribuye a la falta de los siguientes objetivos: i) actualización de IH; ii) actualización de la delimitación de los sitios de humedales atendiendo la clasificación de la CR; iii) frecuencia temporal con periodos de tiempo para el desarrollo de las acciones y alcance de las metas.

La aplicabilidad del modelo de gestión al humedal Urama se basa en la PER (2016-2024) y en la política ambiental de Venezuela, consistiendo en la definición de un área de protección en una unidad de estudio de la cuenca hidrográfica, incluyendo la zona de amortiguación, estructurado por un proceso de planificación compuesto por 5 componentes, 28 variables y 56 atributos denominado modelo para la gestión del humedal Urama.

El modelo para la gestión del humedal Urama, para el proceso de evaluación se basa en variables de impacto para la toma de decisión, como estrategia para la restauración y control del cambio de las características ecológicas, incluye métodos que muestren cambios de UTCT, balance hídrico, niveles de sensibilidad y valoración ambiental de los servicios del humedal, con lo que se procede a formular los objetivos para el PAc.

El modelo para la gestión del humedal Urama, considera entre los objetivos y el PAc, acciones específicas para definir unidades de ordenamiento y el reglamento de uso y manejo para asegurar la sostenibilidad del humedal y el mantenimiento de los servicios del ecosistema, siendo relevante para la conservación de las AP, que los modelos mantengan el monitoreo, el seguimiento y el control de las variables de impacto para incrementar los valores de la gestión.

El modelo para la gestión del humedal Urama, se propone contribuir a la creación de AP como modelo complemento de gestión de sitios de humedales sometidos a la explotación de los recursos naturales que ameritan la restauración, en respuesta a la necesidad de conservación mediante el uso sostenible de los bienes y servicios.

\section{Lista de abreviaturas utilizadas en este artículo}
AL: América Latina
AP: Áreas Protegidas
CA: Características Ecológicas
COP: Conferencia de las Partes

\section{E: Evaluación}

DCE: Descripción de Características Ecológicas

HU: Humedal Urama

IIG: Indicador Implementación de Gestión

IH: Inventario de Humedales

IMGH: Indicador de Modelo de Gestión de Humedales

IMGH_P: Indicador de Modelo de Gestión de Humedales Política

IMGH_CE: Indicador de Modelo de Gestión de Humedales Características Ecológicas

IMGH_PE: Indicador de Modelo de Gestión de Humedales Procesos Ecológicos

IMGH_SE: Indicador de Modelo de Gestión de Humedales Servicios de Ecosistemas

IMGH_E: Indicador de Modelo de Gestión de Humedales Evaluación

IMGH_O: Indicador de Modelo de Gestión de Humedales Objetivos

IMGH_PAc: Indicador de Modelo de Gestión de Humedales Plan de Acción

MHCR: Modelo de Humedales de la Convención de Ramsar

MMC: Matriz Multicriterio

O: Objetivos

PAc: Plan de Acción 
PEMHs: Políticas y Estrategias de Manejo de Humedales

PE: Procesos Ecológicos

PER: Planes Estratégicos de Ramsar

P: Política

SE: Servicios de Ecosistemas

SR: Sitios Ramsar

UTCT: Uso de la Tierra y Cobertura de la Tierra

VTI: Valor Total Integrado

\section{Referencias bibliográficas}

COMTEMA-OLACEFS (2015). Áreas Protegidas América Latina - Auditoría Coordinada. Comisión Técnica Especial de Medio Ambiente de la Organización Latinoamericana y del Caribe de Entidades Fiscalizadoras Superiores. Recuperado de: [https://www.olacefs.com/ resumen-ejecutivo-auditoria-en-areas-protegidas-de-america-latina].

Convención de Ramsar (2015). El Cuarto Plan Estratégico para 2016-2024. Recuperado de: [https://www.ramsar.org/sites/default/files/ documents/library/4th_strategic_plan_2016 _2024_s.pdf].

Costanza, R. D. (1997). The value of the world's ecosystem services and natural capital. Nature, 387, pp. 253-260. Recuperado de: [https://www. biodiversity.ru/programs/ecoservices/library/ common/doc/Costanza_1997.pdf].

Cowardin, L. M., Carter V., Golet, F. C. y LaRoe, E. T. (1979). Classification of wetlands and deepwater habitats of the United States. Washington: Fish and Wildlife Service. Recuperado de:

[https://www.fws.gov/Wetlands/documents/ Classification-of-Wetlands-and-DeepwaterHabitats-of-the-United-States.pdf].

De Groot, R., Stuip, M., Finlayson, M. y Davidson, N. (2007). Valoración de humedales lineamientos para valorar los beneficios derivados de los servicios de los ecosistemas de humedales.
(Informe Técnico de Ramsar Número 3). Recuperado de: [https://www.ramsar.org/sites/ default/files/documents/pdf/lib/lib_rtr03_s. pdf].

Euliss, N. H., Smith, L. M., Wilcox, D. A. y Browne, B. A. (2008). Linking ecosystem processes with wetland management goals: charting a course for a sustainable future. Wetlands, 28(3), pp. 553-562. [DOI:https://doi. org/10.1672/07-154.1].

FGDC (2013) Classification of Wetlands and deepwater habitats of the United States. Federal Geographic Data Committee. Washington: Fish and Wildlife Service. Recuperado de: [https://www.fgdc.gov/standards/projects/ wetlands/nwcs-2013].

Galarza, S. L. (2011). Desarrollo de una herramienta de análisis multicriterio para el soporte de toma de decisiones en el aprovechamiento de aguas de lluvia en el campus de la Pontificia Universidad Javeriana, sede Bogotá (Tesis de maestría). Universidad Pontificia Javeriana, Bogotá, Colombia. Recuperado de: [http://hdl. handle.net/10554/1453].

Gattenlöhner, U., Hammerl-Resch, M. y Jantschke, S. (Eds). (2004). Restauración de Humedales - Manejo Sostenible de Humedales y Lagos Someros. Radolfzell, Alemania: Global Nature Fund (GNF). Recuperado de: [http://ec.europa.eu/environment/life/pro ject $/$ Projects $/$ index.cfm? fuseaction $=$ home . showFile\&rep=file\&fil=LIVING_LAKES_ manual_ES.pdf].

Gibbs, J. P. (2000) Wetland loss and biodiversity conservation. Conservation biology, 14(1), pp. 314-317 [DOI:https://doi.org/10.1046/j. 1523-1739.2000.98608.x].

Hajkowicz, S. C. (2007). A review of multiple criteria analysis for water resource planning and management. Water Resour Manage, 21, pp. 1553-1566 [DOI:https://doi.org/10.1007/ s11269-006-9112-5].

Hammer, D. E. y Kadlec, R. H. (1986) A model for wetland surface water dynamics. Water 
Resources Research, 22(13), pp. 1951-1958 [DOI:https://doi.org/10.1029/WR022i013p 01951].

INPARQUES (2012) Plan Estrátegico 20132019. Instituto Nacional de Parques. Caracas: Gobierno Bolivariano de Venezuela. Recuperado de: [http://musguito.net.ve/anapro/co rredor/proyecto/Plan_Estrategico_de_INPARQUES_2013-2019.pdf].

Kugler, L. (2003). Young conservationists and the future of protected areas worldwide. Communicating. Forestry \& Environmental Studies Publications Series, (18). Recuperado de: [https://elischolar.library.yale.edu/cgi/viewcontent.cgi? article $=1010 \&$ context $=$ fes-pubs].

Márquez, A., Guevara, E. y Rey, D. (2018a). Assessment of land use and land cover change detection using eleven techniques of satellite remote sensing in the Pao river basin, Venezuela. Journal of Remote Sebsing GIS \& Technology, 4(2), pp. 1-70. Recuperado de: [http://matjournals.in/index.php/JORSGT/ article/view/2764].

Marquez, A., Guevara, E. y Rey, D. (2018b). Spatiotemporal forecasting model of water balance variable in the San Diego aquifer, Venezuela. Journal of Remote Sensing GIS \& Technology, 4(3), pp. 1-23. Recuperado de: [http://mat journals.in/index.php/JORSGT/article/view /2864/1971]

Ministerio de Ambiente y Energía de Costa Rica. (2017). Política nacional de humedales 20172030. Recuperado de: [https://minae.go.cr/ recursos/2017/pdf/consulta-linea-politicanacional-humedal.pdf].

Ministerio de Medio Ambiente de España (2001) Plan estratégico español para la conservación y el uso racional de los humedales, en el marco de los ecosistemas acuáticos de que dependen. Recuperado de: [https://www.miteco.gob.es/ es/biodiversidad/temas/ecosistemas-y-conectividad/pan_humedales_tcm30-196686.pdf].

Ministerio de Ambiente de Panamá (2018). Política nacional de humedales de la República de Panamá. Ciudad de Panamá: Ministerior del Medio Ambiente. Recuperado de: [https:// www.undp.org/content/dam/panama/docs/ Documentos_2018/Politica\%20Nacional\%20 Humedales.pdf].

Noon, K. F. (1996). A model of created wetland primary succession. Landscape and Urban planning, 34(2), pp. 97-123 [https://doi. org/10.1016/0169-2046(95)00209-X].

PEQUIVEN (2014) Proyecto desvio de la troncal 3 con conexión a la Autopista Centro Occidental - Estudio de impacto ambiental y sociocultural. Morón: Petroquímica de Venezuela.

República de Colombia. Ministerio del Medio Ambiente (2002). Política nacional para humedales interiores de Colombia, estrategias para su conservación y uso sostenible (primera edición). Bogotá: Ministerio del Medio Ambiente. Recuperado de: [https://www.minambiente. gov.co/images/BosquesBiodiversidadyServiciosEcosistemicos/pdf/Normativa/Politicas/ polit_nal_humedales_int_colombia.pdf].

República de Venezuela (1983). Ley Organica para la Ordenacion del Territorio (Gaceta Oficial $\mathrm{N}^{\circ}$ 3.238 Extraordinario). Recuperado de: [http:// extwprlegs1.fao.org/docs/html/ven 24827 . htm].

Rodríguez, R. (1999). Conservación de humedales en Venezuela: Inventario, diagnóstico ambiental y estrategia. Caracas: Comité Venezolano de la UICN. Recuperado de: [https://portals.iucn. org/library/node/7648].

Rojas, T. (1985). Metodología para la evaluación de áreas sensibles en Venezuela. Revista Geográfica (102), pp. 29-41. Recuperado de: [https:// www.jstor.org/stable/40992497].

Secretaría de la Convención de Ramsar (2010). Manejo de humedales: Marcos para manejar Humedales de Importancia Internacional $y$ otros humedales. Manuales Ramsar para el uso racional de los humedales, 4ta edición, Vol. 18. Gland (Suiza): Secretaría de la Convención de Ramsar. Recuperado de: [https://www.ramsar. 
org/sites/default/files/documents/pdf/lib/ hbk4-18sp.pdf].

Secretaría de la Convención de Ramsar. (2017) Convención Relativa a los Humedales de Importancia Internacional especialmente como Hábitats de Aves Acuáticas. Recuperado de: [https://www.ramsar.org/sites/default/files/ documents/library/current_convention_s.pdf].

Secretaría de la Convención de Ramsar (2018) Resolución XIII.12 Orientaciones para identificar turberas como Humedales de Importancia Internacional (sitios Ramsar) para la regulación del cambio climático mundial como argumento adicional a los criterios existentes de Ramsar. Dubai: Secretaría de Ramsar Convención sobre los humedales. Recuperado de: [https://www. ramsar.org/sites/default/files/documents/ library/xiii.12_identifying_peatlands_ramsar_ sites_s.pdf].
SEMARNAT (2010) Política nacional de humedales. Ciudad de México: Secretaría de Medio Ambiente y Recursos Naturales de México. Recuperado de: [https://agua.org. $\mathrm{mx} / \mathrm{wp}$-content/uploads/2017/07/politicanacional-de-humedales.pdf].

Whigham, D. F. (1999). Ecological issues related to wetland preservation, restoration, creation and assessment. Science of the Total Environment, 240(1-3), pp. 31-40 [DOI:https://doi. org/10.1016/S0048-9697(99)00321-6].

Xie, Z., Xu L., Duan, X. y Xu, X. (2012). Analysis of boundary adjustments and land use policy change-A case study of Tianjin Palaeocoast and Wetland National Natural Reserve, China. Ocean \& coastal management, 56, pp. 56-63. [DOI: https://doi.org/10.1016/j. ocecoaman.2011.06.010]. 